\title{
Sensitivity analyses of a precerebellar neuron model predict a morphologic mechanism for plasticity of neuronal function
}

\author{
Christina M Weaver ${ }^{1 *}$, Aniruddha Yadav ${ }^{2,3}$, Georgi N Gamkrelidze4, Robert Baker ${ }^{4}$ \\ From Nineteenth Annual Computational Neuroscience Meeting: CNS*2010 \\ San Antonio, TX, USA. 24-30 July 2010
}

The vestibuloocular reflex (VOR) provides an amenable model of learning and memory. Neurons from the Area II nucleus of goldfish are necessary for VOR plasticity, carrying an efferent copy of the eye velocity motor command to the cerebellum. Recently we introduced a novel sensitivity analysis method [1] to quantify how dendritic morphology and intrinsic electrical parameters affect neuronal function. This approach also allows precise combinations of active conductances to be predicted that can compensate for a given morphologic change to restore normal function. Here we have extended our method to analyze interactions between explicit pairs of parameters. We reconstructed the morphology and built multi-compartment models of three distinct Area II neurons, followed by an automated parameter optimization to fit each model to a single set of physiological data. Using partial least squares regression to identify sensitivity trends globally across the space of active and morphologic parameters, neuronal outputs relevant to eye velocity integration were found to be driven largely by intracellular calcium concentration near the soma, and voltage attenuation in distal dendrites. We then analyzed the second order sensitivity matrices (Hessians) of perisomatic calcium concentration and distal voltage attenuation, for each morphologic model at two disparate points in parameter space. The results identified sensitive directions, precise combinations of electrical and morphologic parameters that varied output the most, and robust directions that varied output the least. Perturbing the model along the sensitive direction of distal voltage attenuation, which minimally changed perisomatic calcium concentration, was sufficient to induce functional changes that might underlie vestibular plasticity paradigms. Yet, even large perturbations along the robust direction of distal voltage attenuation had little effect on functional output. Our results show that specific, subtle changes of morphology and conductances in the dendrites are sufficient to alter the range of a neuron's firing dynamics. We propose that dendritic morphology plays a significant role in determining the functional flexibility, or robustness, of a neuron.

\section{Acknowledgements}

Supported by NIH grants DC05669, NS13742 and MH071818.

\section{Author details}

${ }^{1}$ Department of Mathematics and Computer Science, Franklin \& Marshall College, Lancaster, PA 17603, USA . ²Department of Neuroscience, Mount Sinai School of Medicine, New York, NY 10029, USA . ${ }^{3}$ Computational Neurobiology and Imaging Center, Mount Sinai School of Medicine, New York, NY 10029, USA . " Department of Physiology and Neuroscience, NYU Langone Medical Center, New York, NY 10016, USA.

Published: 20 July 2010

\section{Reference}

1. Weaver CM, Wearne SL: Neuronal firing sensitivity to morphologic and active membrane parameters. PLoS Comput Biol. 2008, 4:e11.

doi:10.1186/1471-2202-11-S1-P137

Cite this article as: Weaver et al:: Sensitivity analyses of a precerebellar neuron model predict a morphologic mechanism for plasticity of neuronal function. BMC Neuroscience 2010 11(Suppl 1):P137.

* Correspondence: christina.weaver@fandm.edu

1 Department of Mathematics and Computer Science, Franklin \& Marshall College, Lancaster, PA 17603, USA 